\title{
Statins are Associated with Reduced Overall and Cancer-Specific Mortality in Patients Undergoing Radical Cystectomy for Bladder Cancer
}

\author{
Michel D. Wissing, b, ${ }^{\mathrm{a}}$, Ana O'Flaherty ${ }^{\mathrm{a}}$, Alice Dragomir ${ }^{\mathrm{a}}$, Simon Tanguay ${ }^{\mathrm{a}, \mathrm{b}}$, \\ Wassim Kassouf $^{\mathrm{a}}$ and Armen G. Aprikian ${ }^{\mathrm{a}, \mathrm{b}}$ \\ ${ }^{a}$ Department of Surgery, McGill University Health Centre, Montreal, QC, Canada \\ ${ }^{\mathrm{b}}$ Department of Oncology, McGill University, Montreal, QC, Canada
}

Received 13 October 2019

Accepted 20 December 2019

\begin{abstract}
.
BACKGROUND: Existing literature provides contrasting data on statin use and bladder cancer (BC) outcome.

OBJECTIVE: We evaluated whether (chronic) statin use was associated with clinical outcomes in patients with BC undergoing radical cystectomy (RC).

METHODS: Using provincial health administrative databases, we retrospectively identified BC patients undergoing RC in Quebec province in 2000-2015, and collected data from two years before RC until December 2016 or death. We compared patients who chronically used statins before RC to never statin users. Survival analyses were conducted using Kaplan-Meier curves, log-rank tests, and Cox proportional hazards models. Covariates in multivariable analyses were age, sex, Charlson's comorbidity index, year of RC, distance to hospital, hospital type, hospital's and surgeon's annual RC volume, and neoadjuvant chemotherapy.

RESULTS: Our cohort contained 1406 chronic and 1754 never statin users. Five-year overall, BC-specific and recurrencefree survival rates were $40.5 \%$ (95\% confidence interval (95\% CI) $37.8-43.2 \%$ ), $52.8 \%$ (95\% CI $49.8-55.7 \%$ ), and $50.1 \%$ (95\% CI 47.2-53.0\%) for chronic statin users, versus 34.9\% (95\% CI 32.5-37.2\%), 45.5\% (95\%CI 42.9-48.1\%), and 43.4\% (95\% CI 40.9-45.9\%) for never statin users ( $p \leq 0.001)$. In multivariable analyses, hazard ratios (HR) for death, BC-specific deaths and recurrences were 0.83 (95\% CI 0.75-0.91), 0.81 (95\% CI 0.72-0.91), and 0.83 (95\% CI 0.74-0.93) for chronic statin users, respectively. Similar observations were made in patients with diabetes and/or cardiovascular comorbidities $(p \leq 0.001)$. Clinical outcome was not improved in patients who started statins in the year following surgery compared to never statin users $(p>0.4)$.
\end{abstract}

CONCLUSIONS: Chronic statin use is associated with improved clinical outcome in BC patients undergoing RC in Quebec.

Keywords: Urinary bladder neoplasms, survival, Hydroxymethylglutaryl-CoA reductase inhibitors, statin, radical cystectomy

\footnotetext{
${ }^{*}$ Correspondence to: Michel Wissing, Division of Urology, Department of Surgery, McGill University Health Center Research Institute, 1001 Boulevard Decarie, D02.8100, Montreal, Québec, H4A 3J1, Canada. Tel.: +15143986926; Fax: +15148431552; E-mail: michel.wissing@mcgill.ca.
}

\section{INTRODUCTION}

Statins are drugs that lower cholesterol by inhibition of 3-hydroxy-3-methylglutaryl-coenzyme A reductase (HMG-CoA reductase). They are the most commonly prescribed drugs in Canada: $48.4 \%$ of 
individuals aged 65+ used statins in 2016 [1]. Statins and their influence on cancer risk and survival has been widely debated in 21 st century medical literature, with inconclusive findings [2-6]. A potential association between statin use and bladder cancer outcome gained interest in 2006, when a retrospective cohort study was published in the New England Journal of Medicine in which 84 patients with non-muscle invasive bladder cancer had more aggressive disease and a higher rate of tumor progression when taking statins during bacilli Calmette-Guérin therapy [7]. However, since then, various groups failed to confirm an association between statin use and disease-related clinical outcome in patients with non-muscle invasive bladder cancer [8-12].

Fewer studies have evaluated statins in patients with muscle-invasive bladder cancer. In a cohort of patients with muscle-invasive bladder cancer who underwent transurethral resection of the bladder followed by chemoradiotherapy, statin users had higher local disease control in univariable analyses, but this difference was eliminated when adjusting for known prognostic factors [13]. The only cohort study evaluating statin use in patients with muscle-invasive bladder cancer undergoing radical cystectomy (RC) found that statin users had higher rates of disease recurrence and cancer-specific mortality in univariable analyses, but clinical outcome did not differ in multivariable analyses [14].

Large, high-quality studies are needed to evaluate a potential protective or harmful effect of statins on clinical outcome in patients with muscle-invasive bladder cancer. Therefore, we evaluated statin use in a large cohort of bladder cancer patients undergoing $\mathrm{RC}$ in the Canadian province of Quebec.

\section{PATIENTS AND METHODS}

\section{Study design, data acquisition and study population}

This retrospective study was approved by the research ethics board of the McGill University Health center, and is annually renewed (project number 2014-1059; latest renewal August 31st, 2018). It follows international guidelines such as the Declaration of Helsinki. Our cohort consists of all patients who underwent RC for bladder cancer in Quebec between 2000 and 2015, as registered by the universal health care provider provincial administrative databases [15]. Quebec administrative agencies collect data on all medical procedures performed in public medical centers across Quebec, all hospital and emergency room admissions, intensive care unit stays, all medication prescribed in Quebec outpatient pharmacies to patients covered by the Public Prescription Drug Insurance Plan (universal coverage), and all deaths in Quebec (including date and cause of death). Since RC in Quebec is only conducted in public hospitals, data provided by provincial authorities included essentially all RC in Quebec. The collected database contained anonymized individual patient medical information from two years before RC to death or December 31st, 2016. As such, no informed consent was required.

Selection of our cohort has been described previously [15]. In summary, after several validity checks, we included all patients who had a RC conducted by a urologist in a public hospital in the province of Quebec between January 1st, 2000 and December 31st, 2015. All selected RC were performed for the treatment of bladder cancer $(n=4450)$. Patients who were not enrolled in the Quebec Public Prescription Drug Insurance Plan were excluded $(n=708$ $(15.9 \%)$ ), since we could not obtain information on their medication use.

\section{Data analyses}

Chronic statin users were defined as patients who had $\geq 1$ statin prescription before RC and who had statin prescriptions $\geq 365$ days between the first and last prescription in our database. A prescription was registered in our database if the patient picked up his/her medication at an outpatient pharmacy in Quebec. Controls never obtained statins at a pharmacy in the two years prior to RC until the most recent followup. Sensitivity analyses were conducted by limiting both groups to patients who had a diagnostic code for diabetes mellitus or cardiovascular disease (congestive heart failure, myocardial infarction, peripheral vascular disease, and/or a cerebrovascular accident) during their treatment trajectory. Furthermore, we compared patients who had their first statin prescription in the year after surgery to never statin users. In order to eliminate immortal time bias in this analysis, we restricted both groups to patients with a survival of $\geq 1$ year.

Covariates included age, sex, and Charlson's comorbidity index. We also included all variables that predicted survival in our previously published analyses, namely the year of surgery, distance to the hospital, hospital type (academic/non-academic), hospital and surgeon's annual RC volume, and 
administration of neoadjuvant chemotherapy. Age and Charlson's comorbidity index were analyzed as continuous variables, sex as a categorical variable. The Charlson's comorbidity index was determined based on filed diagnostic codes, and on medication specifically used for certain diseases [16, 17]. Patients could have an index between two (all patients had a solid tumor, which is two points) and 33. Year of surgery was included as a binary categorical variable, as we previously observed a difference in survival between patients treated in 2000-2009 and those treated in 2010-2015 [15]. We used Google maps to calculate the driving distance between the center of a patient's postal code and the hospital of surgery (continuous variable) [15]. Academic hospitals include all centers that train residents. Hospital and surgeon annual RC volumes were calculated by dividing the total number of RC by the years between the first and last RC of that surgeon/hospital recorded in our database, and were analyzed as continuous variables.

Follow-up time was calculated using the reverse Kaplan-Meier method [18]. Time to recurrence was defined as the time between RC and first administration of chemo- or radiotherapy after surgery, or death due to bladder cancer. In order to exclude patients who had neoadjuvant chemo- or radiotherapy, or salvage therapy due to incomplete removal of the tumor, we only considered chemo- or radiotherapy administered $\geq 90$ days after postoperative hospital discharge.

Overall survival was calculated as the time between $\mathrm{RC}$ and death or last follow-up. The definition of bladder cancer-specific deaths has been prescribed previously [15]. In brief, all death causes with ICD-10 codes for genitourinary cancers were included, unless the diagnosis specified a different genitourinary cancer (e.g., prostate cancer). For bladder cancer-specific mortality, we excluded patients who died $\leq 90$ days after RC, to exclude patients who died from the direct consequences of surgery instead of the cancer; these patients were included in overall survival calculations. Patients who died from other causes than bladder cancer were censored at the time of death.

\section{Statistical analyses}

All statistical analyses were conducted using Stata (v15.1, Statacorp, College Station, Texas, USA). Categorical baseline characteristics were compared between chronic and never statin users using Chisquare tests. All continuous baseline characteristics were non-normally distributed, as determined by Shapiro-Wilk tests. Therefore, continuous variables were compared using Wilcoxon rank-sum tests, and summarized as the median with interquartile range.

Survival analyses were conducted using the Kaplan-Meier method [19]. Log-rank tests were used to calculate whether survival curves differed significantly between patient groups. Uni- and multivariable Cox proportional hazard models were used to calculate hazard ratios (HR) for overall, bladder cancer-specific and recurrence-free survival [20]. All covariates (i.e., statin use, age, sex, comorbidities,

Table 1

Baseline characteristics of the whole cohort, patients who chronically used statins before radical cystectomy, and patients who never used statins

\begin{tabular}{|c|c|c|c|c|}
\hline & All patients & never statin users & chronic statin users & $P$-value ${ }^{1}$ \\
\hline Number of patients & 3742 & 1754 & 1406 & \\
\hline Age, median (IQR) & $70(64-76)$ & $70(62-76)$ & $72(67-77)$ & $<0.001$ \\
\hline Sex & & & & $<0.001$ \\
\hline - Male & $2827(75.5 \%)$ & $1244(70.9 \%)$ & $1113(79.2 \%)$ & \\
\hline - Female & $915(24.5 \%)$ & $510(29.1 \%)$ & $293(20.8 \%)$ & \\
\hline Charlson's comorbidity index & $7(5-8)$ & $6(5-8)$ & $7(6-9)$ & $<0.001$ \\
\hline- Index $\geq 10$ & $500(13.4 \%)$ & $125(7.1 \%)$ & $274(19.5 \%)$ & \\
\hline - Range & $2-18$ & $2-18$ & $2-15$ & \\
\hline Year of surgery & & & & $<0.001$ \\
\hline$-2000-2009$ & $2203(58.9 \%)$ & $1138(64.9 \%)$ & $632(45.0 \%)$ & \\
\hline$-2010-2015$ & $1539(41.1 \%)$ & $616(35.1 \%)$ & $774(55.0 \%)$ & \\
\hline Hospital type, academic & $1886(50.4 \%)$ & $904(51.5 \%)$ & $711(50.6 \%)$ & 0.59 \\
\hline Distance to hospital in km, median (IQR) & $19.9(7.4-77.2)$ & $19.0(6.9-66.5)$ & $20.6(7.9-87.5)$ & 0.029 \\
\hline Hospital RC volume per active year, median (IQR) & $13.4(7.8-32.2)$ & $13.4(6.6-32.2)$ & $13.8(8.9-32.2)$ & 0.003 \\
\hline Surgeon RC volume per active year, median (IQR) & $6.7(4.7-13.1)$ & $6.7(4.1-13.1)$ & $8.1(4.7-13.1)$ & 0.005 \\
\hline Neoadjuvant chemotherapy & $208(5.6 \%)$ & $108(6.2 \%)$ & $75(5.3 \%)$ & 0.33 \\
\hline
\end{tabular}

Values display the median with interquartile range for continuous variables, and the absolute patient number with percentage for categorical variables. ${ }^{1} P$-values were determined using Wilcoxon rank sum tests and Chi-square tests for continuous and categorical variables, respectively. 
year of surgery, distance to hospital, hospital type, hospital's and surgeon's RC volume, and administration of neoadjuvant chemotherapy) were included in multivariable models. In an additional multivariable model, we adjusted for aforementioned variables plus chronic aspirin and metformin use (defined as aspirin and metformin prescriptions for more than 365 days). Patients with missing data were excluded from multivariable analyses.

\section{RESULTS}

In total, 3742 patients underwent RC for bladder cancer in Quebec in 2000-2015 and had information on medication use available. Median age was 70 years, $75.5 \%$ was male, and the median comorbidity index was seven (Table 1). Approximately half of the patients $(n=1886,50.4 \%)$ had RC in an academic hospital, and 208 patients $(5.6 \%)$ had neoadjuvant chemotherapy. Of the 1988 patients who had $\geq 1$ prescription for statins in our database, 1512 patients $(76.1 \%)$ had $\geq 1$ prescription prior to $\mathrm{RC}$. We only included these patients to eliminate immortal time bias. Of these 1512 patients, 1406 patients (93.0\%) had $\geq 365$ days between the first and last statin prescription (chronic statin users), and were compared to patients who never obtained statins $(n=1754)$. Chronic statin users were older (median age 72 versus 70 years, $p<0.001)$, male $(79.2 \%$ versus $70.9 \%, p<0.001$ ), and had more comorbidities (median seven versus six, $p<0.001$ ). Chronic statin users were treated more recently $(55.0 \%$ versus $35.1 \%$ treated in 2010-2015, $p<0.001$ ), in hospitals slightly further away from their residence (median $20.6 \mathrm{~km}$ versus $19.0 \mathrm{~km}, p=0.029$ ) by higher volume surgeons (8.1 versus 6.7 RC per year, $p=0.005$ ).

Median follow-up was 7.9 years (95\% confidence interval (95\%CI) 7.6-8.4 years). Of the 1361 patients who had not died by December 2016, 80 (5.9\%) had their latest follow-up in 2015 or earlier (lost to follow-up). Five-year overall survival rates were $40.5 \%$ (95\%CI $37.8-43.2 \%$ ) and $34.9 \%$ (95\%CI 32.5-37.2\%) for chronic and never statin users, respectively ( $p=0.001$; Fig. 1A). Similarly, five-year bladder cancer-specific survival rates were $52.8 \%(95 \% \mathrm{CI} 49.8-55.7 \%)$ and $45.5 \%$ (95\% CI $42.9-48.1 \%, p<0.001$; Fig. 1B), and five-year recurrence-free survival rates were $50.1 \%(95 \% \mathrm{CI}$ $47.2-53.0 \%$ ) and $43.4 \%$ (95\%CI $40.9-45.9 \%$ ), respectively $(p<0.001$; Fig. 1C). In multivariable models (Table 2), chronic statin use (HR 0.83, 95\% CI
0.75-0.91), age (HR 1.22 per five-year increment, 95\%CI 1.18-1.26), and the Charlson's comorbidity index (HR 0.95 per point increase, 95\% CI $0.92-0.97$ ) predicted overall survival. These variables also predicted bladder cancer-specific survival and recurrence-free survival, the HR for chronic statin users being 0.81 (95\% CI 0.72-0.91) and 0.83 (95\% CI 0.74-0.93), respectively. Although not statistically significant, bladder cancer-specific survival might also be worse for patients with a longer distance

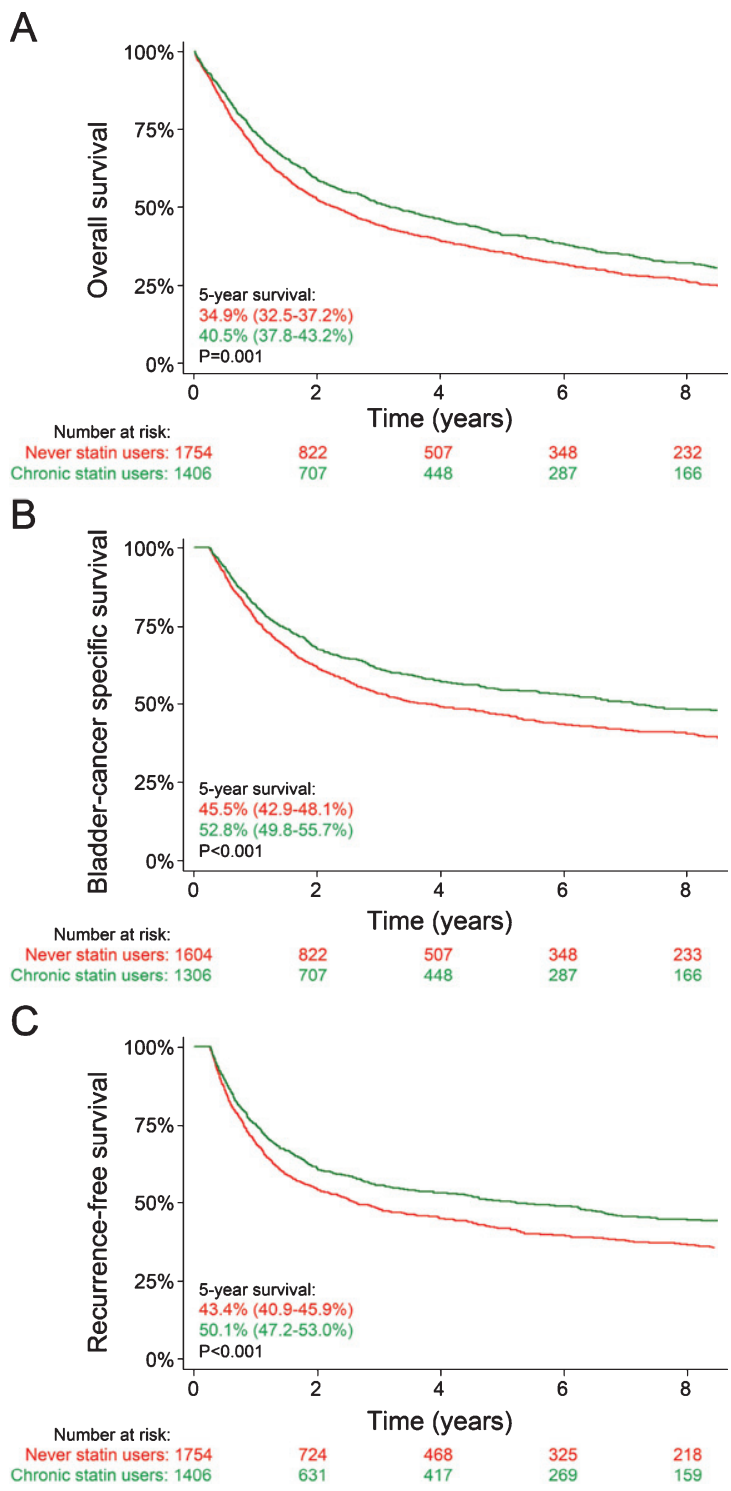

Fig. 1. Overall survival (A), bladder cancer-specific survival (B), and time to recurrence $(C)$ in patients who chronically used statins prior to surgery (green line) and never statin users (red line). Values depict five-year survival rates with their respective $95 \%$ confidence intervals. $P$-values were calculated using log-rank tests. 


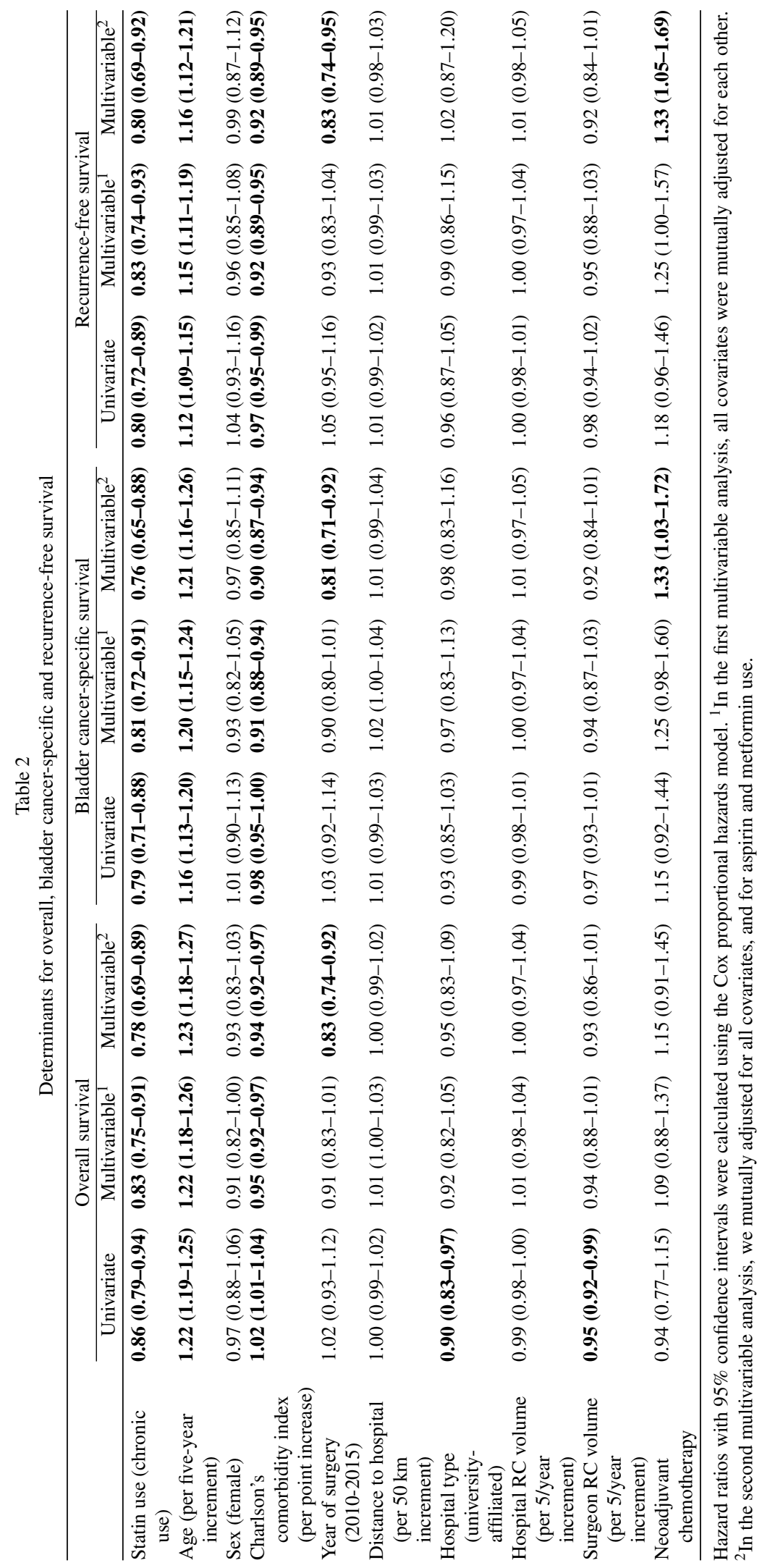


to the hospital of surgery $(p=0.051)$. Addition of chronic aspirin and metformin use as covariates in the multivariable model did not change the findings that chronic statin use predicted overall (HR 0.78, 95\%CI 0.69-0.89), bladder cancer-specific (HR 0.76, 95\%CI 0.65-0.88) and recurrence-free survival (HR $0.80,95 \%$ CI $0.69-0.92$ ).

Considering that most chronic statin users would have diabetes mellitus and/or cardiovascular comorbidities, we conducted a subgroup analysis including all patients with these comorbidities (supplementary table 1). These 968 chronic statin users were still more frequently male $(81.3 \%$ versus $69.7 \%$, $p<0.001$ ) than the 680 controls, had a higher comorbidity index (median eight versus seven, $p<0.001$ ) due to a higher frequency of diabetes $(63.2 \%$ versus $49.3 \%, p<0.001)$, myocardial infarctions $(27.1 \%$ versus $18.4 \%, p<0.001$ ), and peripheral vascular disease $(24.0 \%$ versus $16.8 \%, p<0.001)$, and had more recent surgery $(52.4 \%$ versus $28.4 \%$ having surgery between 2010 and 2015, $p<0.001$ ) in hospitals further away from their residence (20.2 versus $16.2 \mathrm{~km}$, $p=0.020$ ) by higher volume surgeons ( 7.3 versus 6.7 surgeries/year, $p=0.041)$ in higher volume hospitals (13.4 versus 12.8 surgeries/year, $p=0.004$ ). Overall survival, bladder cancer-specific survival and recurrence-free survival were significantly higher in chronic statin users (log-rank tests: $p<0.001$; supplementary figure 1 ), also after adjusting for potential confounders, with adjusted HR of 0.77 (95\% CI 0.68-0.88), 0.75 (95\% CI 0.64-0.88) and 0.77 (95\% CI 0.66-0.90), respectively (supplementary table 2 ).
Next, we evaluated whether patients who started statin therapy after RC had better clinical outcomes. Ninety-one patients started statin therapy in the year following surgery; the control group consisted of 1168 never statin users who survived $\geq 1$ year (Table 3). Patients who started statins after surgery were more frequently male $(82.4 \%$ versus $70.6 \%$, $p=0.016$ ), had a higher comorbidity index (median seven versus six, $p<0.001)$, and were less frequently treated in academic hospitals $(38.5 \%$ versus $51.8 \%$, $p=0.014)$. Outcome between groups were similar: five-year overall survival rates were $51.3 \%$ versus $52.2 \%(P=0.57)$, five-year bladder cancer-specific survival rates were $62.8 \%$ versus $59.9 \%(p=0.27)$, and five-year recurrence-free survival rates were $54.7 \%$ versus $52.7 \%$ ( $p=0.49$; Fig. 2). Similarly, in multivariable analyses, HR for death, bladder cancer-specific deaths, and recurrences were 0.95 (95\%CI 0.69-1.30), 0.87 (95\%CI 0.59-1.27), and 0.95 (95\%CI 0.67-1.33; Table 4).

\section{DISCUSSION}

This is the first study to show that chronic statin use may improve clinical outcomes of bladder cancer patients undergoing RC. In line with our findings, preclinical data suggested that statins may improve bladder cancer outcome via various mechanisms of action, including antiproliferative, pro-apoptotic, anti-inflammatory, and antioxidant activity [21-23]. In other urogenital cancers, statins also inhibited angiogenesis and invasion/metastasis [24].

Table 3

Baseline characteristics of patients who started statin therapy within a year after radical cystectomy and never-users who survived at least 1 year post-surgery

\begin{tabular}{|c|c|c|c|}
\hline & Never statin users & Statin users & $P$-value ${ }^{1}$ \\
\hline Number of patients & 1168 & 91 & \\
\hline Age, median (IQR) & $68(61-75)$ & $66(64-73)$ & 0.57 \\
\hline Sex & & & 0.016 \\
\hline - Male & $825(70.6 \%)$ & $75(82.4 \%)$ & \\
\hline - Female & $343(29.4 \%)$ & $16(17.6 \%)$ & \\
\hline Charlson's comorbidity index & $6(5-8)$ & $7(6-9)$ & $<0.001$ \\
\hline- Index $\geq 10$ & $91(7.8 \%)$ & $10(11.0 \%)$ & \\
\hline - Range & $2-15$ & $2-12$ & \\
\hline Year of surgery & & & 0.06 \\
\hline$-2000-2009$ & $756(64.7 \%)$ & $50(54.9 \%)$ & \\
\hline$-2010-2015$ & $412(35.3 \%)$ & $41(45.1 \%)$ & \\
\hline Hospital type, academic & $605(51.8 \%)$ & $35(38.5 \%)$ & 0.014 \\
\hline Distance to hospital in km, median (IQR) & $18.2(7.1-63.6)$ & $18.8(8.0-57.3)$ & 0.94 \\
\hline Hospital RC volume per active year, median (IQR) & $13.4(7.8-32.2)$ & $12.8(5.3-16.1)$ & 0.052 \\
\hline Surgeon RC volume per active year, median (IQR) & $6.7(4.7-13.1)$ & $6.7(4.7-13.1)$ & 0.81 \\
\hline Neoadjuvant chemotherapy & $75(6.4 \%)$ & $4(4.4 \%)$ & 0.44 \\
\hline
\end{tabular}

Values display the median with interquartile range for continuous variables, and the absolute patient number with percentage for categorical variables. ${ }^{1} P$-values were determined using Wilcoxon rank sum tests for continuous variables and Chi-square tests for categorical variables. 
A

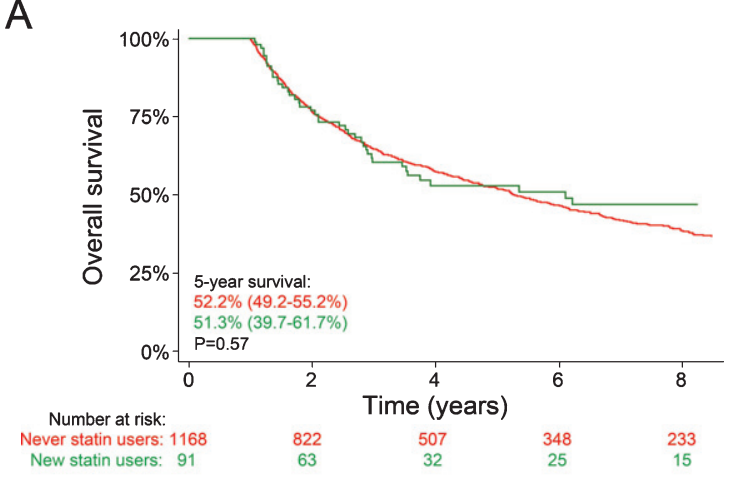

B

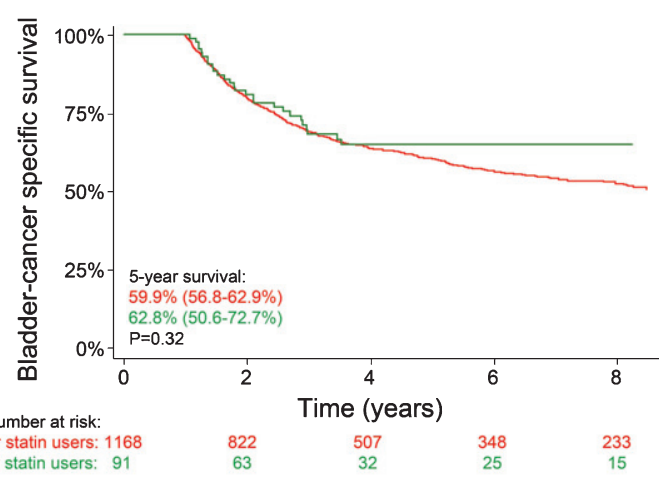

C

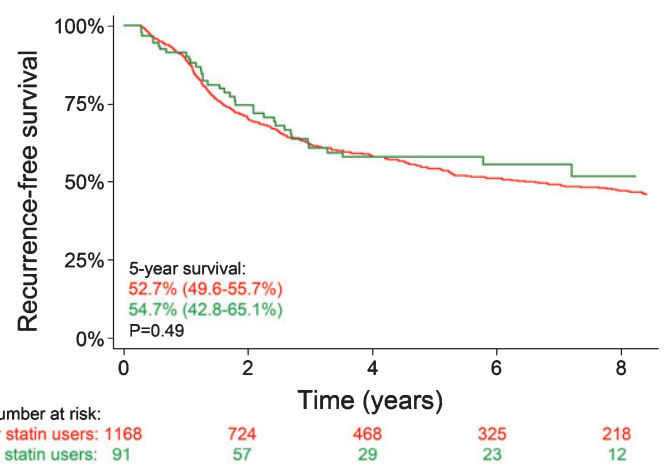

Fig. 2. Overall survival (A), bladder cancer-specific survival (B), and time to recurrence $(\mathrm{C})$ in patients who started statins in the year following surgery (green line) and never statin users (red line) who survived at least one year post-surgery. Values depict fiveyear survival rates with their respective $95 \%$ confidence intervals. $P$-values were calculated using log-rank tests.

Only one other clinical study evaluated statin use in a similar patient population. This study did not find significant differences in disease recurrence or cancer-specific mortality [14]. They had access to pathology reports, thus being able to adjust for differences in tumor stage and grade. Potentially, discrepancies between our findings could be explained if statin users have less advanced tumors at surgery; however, Da Silva and colleagues did not observe a difference in tumor stage and grade in statin users. Alternatively, the published study may have been underpowered due to fewer patients and a shorter follow-up. They stratified by statin use at the time of RC, regardless of medical history, while we limited our cases to chronic ( $\geq 1$ year of statin use) and never statin users. Furthermore, their reported other-cause mortality rate is significantly elevated compared to a general age- and sex-matched population. Considering that patients with a short expected life span (e.g., due to comorbidities) would generally not be considered for RC, one would have expected relatively similar other-cause mortality rates. Hence, their results suggest underreporting of bladder cancer-related deaths. Indeed, misclassification occurs frequently when using death certificates to determine the death cause to estimate cancer-specific mortality [25-28]. To prevent underreporting of cancer-related deaths, we used a relatively liberal definition of cancer-specific mortality; in our population, other-cause mortality is only slightly higher than the mortality rate in a general ageand sex-matched population, which may be explained by differences in smoking rates.

Previously, researchers have suggested that the benefit of statin use may have been confounded by concomitant aspirin use [29, 30]. After adjusting for chronic aspirin and metformin use in our multivariable analyses, statins still had a protective effect: HR for death, cancer-specific deaths and recurrences in chronic statin users were 0.78 (95\% CI 0.69-0.89), 0.76 (95\%CI 0.65-0.88), and 0.80 (95\% CI 0.69-0.92), respectively.

Our analyses eliminated biases that are common in studies evaluating an association between statin use and cancer outcome [6]. We eliminated immortal time bias: chronic statin users had initiated statin therapy before $\mathrm{RC}(t=0)$, and when evaluating patients who initiated statin therapy postoperatively, we limited our analyses to patients who survived for $\geq 1$ year [6]. Our study is the largest on this topic, reducing the risk that our cohort was underpowered to detect significant differences.

However, our results are inevitably subject to biases. Patients with a lower comorbidity index had worse clinical outcomes. This appears contradictory, but can be explained as patients with severe comorbidities tend to have more stringent selection criteria for RC. Inevitably, statin users had a higher comorbidity index. Our non-statin user group would likely include patients unwilling or unable to use statins, for example due to side effects; such patients might have 
Table 4

Initiation of statin therapy after radical cystectomy does not improve overall, bladder cancer-specific or recurrence-free survival

\begin{tabular}{lccc}
\hline & Univariate & Multivariable $^{1}$ & Multivariable $^{2}$ \\
\hline Overall survival & $0.92(0.68-1.24)$ & $0.95(0.69-1.30)$ & $0.75(0.46-1.22)$ \\
Bladder cancer-specific survival & $0.81(0.55-1.18)$ & $0.87(0.59-1.27)$ & $0.65(0.36-1.21)$ \\
Recurrence-free survival & $0.89(0.63-1.24)$ & $0.95(0.67-1.33)$ & $0.84(0.50-1.42)$ \\
\hline
\end{tabular}

Hazard ratios with $95 \%$ confidence intervals were calculated using the Cox proportional hazards model. ${ }^{1}$ Adjusted for age, sex, Charlson's comorbidity index, year of surgery, type of hospital (academic/non-academic), distance to the hospital, hospital and surgeon's radical cystectomy volume, and neoadjuvant chemotherapy. ${ }^{2}$ Adjusted for age, sex, Charlson's comorbidity index, year of surgery, type of hospital (academic/non-academic), distance to the hospital, hospital and surgeon's radical cystectomy volume, neoadjuvant chemotherapy, chronic aspirin use, and chronic metformin use.

a worse clinical outcome in general. Healthy user bias applies to every treatment studied. On the other hand, we reduced selection bias by including all patients undergoing RC for bladder cancer in Quebec, thereby including patients going to non-academic hospitals and others who would generally not be recruited for studies.

Our database lacks important variables. Pathology data is unavailable in the administrative dataset, and due to privacy legislation, Quebec provincial agencies do not allow patient identification to collect these data. Statin users may have had less advanced disease when undergoing radical cystectomy as compared to non-statin users. Previous studies did not identify bladder cancer staging as a confounder though, as there was no association between statin use and bladder cancer staging in patients undergoing RC [14]. We were also unable to evaluate a potential dosedependent association between statin use and bladder cancer outcome, as we only had access to medication purchases for up to two years before RC. We did not have any laboratory values (e.g., lipid profile) of patients either. While we know that patients bought the medication in an outpatient pharmacy, we cannot verify that they were compliant. On the other hand, it is unlikely that a patient would buy statins for at least one year, without taking the medication. Smoking history was unavailable. Finally, few patients initiated statins postoperatively, resulting in low statistical power for these analyses.

The clinical importance of this study is that on the contrary to previous reports [7, 14], we do not find a negative association between statin use and bladder cancer outcome. Hence, statins can be safely continued in bladder cancer patients. Furthermore, the observed positive associations are hypothesis generating. We think our results justify a large, independent cohort study or randomized controlled trial. Preferably, such a study would include data on pathology results, detailed information on statin exposure (duration and dose), and a larger group of patients who initiate statin therapy after surgery. One phase 2 trial evaluating simvastatin and metformin in bladder cancer patients was started in 2015 (NCT02360618) with the results not yet reported. Future studies may also evaluate whether a diet lowering cholesterol impacts bladder cancer outcome.

In conclusion, chronic statin use may improve outcomes of bladder cancer patients undergoing RC. Our findings are in line with a potential protective effect of statins in preclinical bladder cancer studies and beneficial clinical effects reported in other cancers $[4,31]$. Validation of these results in an independent, large cohort or randomized clinical trial will be necessary.

\section{ACKNOWLEDGMENTS}

The authors wish to thank the RAMQ, MSSS, ISQ, and CAI for allowing analyses of the Quebec provincial registry data. We would also like to thank Dr. Fabiano Santos for providing the SAS programming codes he used for analyses of a previous version of the bladder cancer cohort.

\section{FUNDING}

MW was funded by a Fellowship Award from the Canadian Institutes of Health Research (CIHR), McGill University, the Richard Tomlinson Chair in Urology at McGill University held by Armen Aprikian, and the McGill University Health Centre. The funding institutions did not have any influence on the design and conduct of the study; collection, management, analysis, and interpretation of the data; and preparation, review, or approval of the manuscript.

\section{AUTHOR CONTRIBUTIONS}

MDW, WK and AGA contributed to the study conceptualization and design; MDW and AGA contributed to the acquisition of data; MDW, AOF and 
AGA contributed to the analysis of data; MDW, AOF, AD, ST, WK and AGA contributed to the interpretation of data; MDW and AOF drafted the manuscript; AD, ST, WK and AGA critically revised the manuscript.

\section{CONFLICT OF INTEREST}

The authors do not have conflicts of interest to declare.

\section{SUPPLEMENTARY MATERIAL}

Comparisons of patients who chronically used statins before radical cystectomy and those who never used statins, limited to patients with diabetes and/or cardiovascular disease.

\section{REFERENCES}

[1] Canadian Institute for Health Information, Drug use among seniors in Canada, 2016 [Internet]. 2016 [cited 2019 Jan 30]; Available from: https://www.cihi.ca/sites/ default/files/document/drug-use-among-seniors-2016-enweb.pdf.

[2] Graaf MR, Beiderbeck AB, Egberts AC, Richel DJ, Guchelaar HJ. The risk of cancer in users of statins. J Clin Oncol. 2004;22(12):2388-94.

[3] Coogan PF, Rosenberg L, Strom BL. Statin use and the risk of 10 cancers. Epidemiology (Cambridge, Mass). 2007;18(2):213-9.

[4] Voorneveld PW, Reimers MS, Bastiaannet E, Jacobs RJ, van Eijk R, Zanders MMJ, et al. Statin use after diagnosis of colon cancer and patient survival. Gastroenterology. 2017;153(2):470-9.e4.

[5] Jian-Yu E, Graber JM, Lu SE, Lin Y, Lu-Yao G, Tan XL. Effect of Metformin and Statin use on survival in pancreatic cancer patients: A systematic literature review and metaanalysis. Current medicinal chemistry. 2018;25(22):2595607.

[6] Emilsson L, Garcia-Albeniz X, Logan RW, Caniglia EC, Kalager M, Hernan MA. Examining bias in studies of statin treatment and survival in patients with cancer. JAMA Oncol. 2018;4(1):63-70.

[7] Hoffmann P, Roumeguere T, Schulman C, van Velthoven R. Use of statins and outcome of BCG treatment for bladder cancer. N Engl J Med. 2006;355(25):2705-7.

[8] Kamat AM, Wu X. Statins and the effect of BCG on bladder cancer. N Engl J Med. 2007;356(12):1276; author reply -7 .

[9] Skolarus TA, Lee EW, Virgo KS, Katz MD, Hudson MA, Kibel AS, et al. Intravesical bacille Calmette-Guerin therapy for non-muscle-invasive bladder cancer: Effects of concurrent statin therapy. Journal of the American College of Surgeons. 2009;209(2):248-53.

[10] Crivelli JJ, Xylinas E, Kluth LA, da Silva RD, Chrystal J, Novara G, et al. Effect of statin use on outcomes of nonmuscle-invasive bladder cancer. BJU Int. 2013;112(2):E412 .
[11] Luo Y, She DL, Xiong H, Fu SJ, Yang L. The prognostic effect of statin use on urologic cancers: An updated Meta-analysis of 35 observational studies. Medicine. 2015;94(36):e1523.

[12] Richard PO, Ahmad AE, Bashir S, Hamilton RJ, Nam RK, Leao R, et al. Effect of statins as a secondary chemopreventive agent among individuals with non-muscle-invasive bladder cancer: A population-based analysis. Urol Oncol. 2017;35(6):342-8.

[13] Tsai HK, Katz MS, Coen JJ, Zietman AL, Kaufman DS, Shipley WU. Association of statin use with improved local control in patients treated with selective bladder preservation for muscle-invasive bladder cancer. Urology. 2006;68(6):1188-92.

[14] da Silva RD, Xylinas E, Kluth L, Crivelli JJ, Chrystal J, Chade D, et al. Impact of statin use on oncologic outcomes in patients with urothelial carcinoma of the bladder treated with radical cystectomy. J Urol. 2013;190(2):487-92.

[15] Wissing MD, Santos F, Zakaria AS, O'Flaherty A, Tanguay S, Kassouf W, et al. Short- and long-term survival has improved after radical cystectomy for bladder cancer in Quebec during the years 2000-2015. J Surg Oncol. 2019.

[16] Charlson M, Szatrowski TP, Peterson J, Gold J. Validation of a combined comorbidity index. Journal of clinical epidemiology. 1994;47(11):1245-51.

[17] Charlson ME, Pompei P, Ales KL, MacKenzie CR. A new method of classifying prognostic comorbidity in longitudinal studies: development and validation. Journal of Chronic Diseases. 1987;40(5):373-83.

[18] Schemper M, Smith TL. A note on quantifying follow-up in studies of failure time. Control Clin Trials. 1996;17(4):3436.

[19] Kaplan EL, Meier P. Nonparametric estimation from incomplete observations. Journal of the american statistical association. 1958;53(282):457-81.

[20] Cox DR. Regression Models and life-tables. Journal of the Royal Statistical Society Series B (Methodological). 1972;34(2):187-220.

[21] Kamat AM, Nelkin GM. Atorvastatin: A potential chemopreventive agent in bladder cancer. Urology. 2005;66(6): 1209-12.

[22] Parada B, Reis F, Pinto A, Sereno J, Xavier-Cunha M, Neto P, et al. Chemopreventive efficacy of Atorvastatin against nitrosamine-induced rat bladder cancer: Antioxidant, anti-proliferative and anti-inflammatory properties. International Journal of Molecular Sciences. 2012;13(7): 8482-99.

[23] Wang G, Cao R, Wang Y, Qian G, Dan HC, Jiang W, et al. Simvastatin induces cell cycle arrest and inhibits proliferation of bladder cancer cells via PPARgamma signalling pathway. Scientific Reports. 2016;6:35783.

[24] Pon D, Abe A, Gupta EK. A review of statin use and prostate cancer. Current Atherosclerosis Reports. 2015;17(2):474.

[25] Percy C, Stanek E, 3rd, Gloeckler L. Accuracy of cancer death certificates and its effect on cancer mortality statistics. Am J Public Health. 1981;71(3):242-50.

[26] Kircher T, Nelson J, Burdo H. The autopsy as a measure of accuracy of the death certificate. N Engl J Med. 1985;313(20):1263-9.

[27] Turner EL, Metcalfe C, Donovan JL, Noble S, Sterne JA, Lane JA, et al. Contemporary accuracy of death certificates for coding prostate cancer as a cause of death: Is reliance on death certification good enough? A comparison with blinded review by an independent cause of death evaluation committee. Br J Cancer. 2016;115(1):90-4. 
[28] Wissing MD, Greenwald ZR, Franco EL. Improving the reporting of cancer-specific mortality and survival in research using cancer registry data. Cancer Epidemiol. 2019;59:232-5.

[29] Ozgur BC, Sarici H, Yuceturk CN. Re: Impact of statin use on oncologic outcomes in patients with urothelial carcinoma of the bladder treated with radical cystectomy: R. D. da Silva, E. Xylinas, L. Kluth, J. J. Crivelli, J. Chrystal, D. Chade, G. B. Guglielmetti, A. Pycha, Y. Lotan, P. I. Karakiewicz, M. Sun, H. Fajkovic, M. Zerbib, D. S.
Scherr and S. F. Shariat J Urol 2013;190:487-492. J Urol. 2014;192(2):618-9.

[30] Lyon TD, Frank I, Shah PH, Tarrell R, Cheville JC, Karnes $\mathrm{RJ}$, et al. The association of aspirin use with survival following radical cystectomy. J Urol. 2018;200(5):1014-21.

[31] Larsen SB, Dehlendorff C, Skriver C, Dalton SO, Jespersen CG, Borre M, et al. Postdiagnosis statin use and mortality in danish patients with prostate cancer. J Clin Oncol. 2017;35(29):3290-7. 